\title{
Low-Cost Power Systems Metrology Laboratory based on Raspberry Pi
}

\author{
Quijano Cetina, R. ${ }^{1}$, Roscoe, A. J. ${ }^{1}$ and A. Castillo Atoche ${ }^{2}$ \\ ${ }^{1}$ Dept. of Electronic and Electrical Engineering, University of Strathclyde, Glasgow, UK renan.quijano-cetina@strath.ac.uk \\ ${ }^{2}$ Department of Mechatronics, Universidad Autonóma de Yucatán, Mérida, Yuc., México, acastill@correo.uady.mx
}

\begin{abstract}
In this paper, a low-cost Power Systems Metrology Laboratory, based on the Raspberry Pi board and the ADE7878 energy metering IC is presented. The designed experimental platform is intended for developing skills among the undergraduate students of the University of Strathclyde, Glasgow, and the Autonomous University of Yucatan, Mexico. A series of exercises have been developed in order to measure different Power quantities and to evaluate different definitions of such quantities to observe how the accuracy of the measurements is affected, particularly, when non-sinusoidal conditions exist. The system is capable to perform measurements of single or three-phase, employing current transformers. A set of Simulink blocks is ready to use for the students, including PMU algorithms. The proposed laboratory facilitate the evaluation of new algorithms and functions, developed by the students.
\end{abstract}

Index Terms-Power Systems Metrology, Power Quality, Raspberry Pi, PMU.

\section{INTRODUCTION}

The metrology requirements for the upcoming smart grid deployment are moving faster to more accurate measurements. This is particularly important when the conditions of the electrical grid are non-sinusoidal. When power quality disturbances like harmonics, inter-harmonics and unbalance exist in the electrical grid, the error of electricity meters could be significant, depending on the design of the meter and the measurement techniques adopted (i.e. transducer frequency response, measurement algorithm, etc.) $[1]$.

The power quantities definitions on the IEEE-1459 standard [2] addressed not only sinusoidal waveforms, as previous standards done, but also non-sinusoidal and unbalanced conditions. However, such definitions are not implemented by many metering devices available on the market (i.e. they may implement Budeanu or Fryze power definitions). In this regard, a deep understanding of the advantages and disadvantages of using different electrical quantities definitions is necessary, in order to address the needs of the metrology for smart grids.

For energy consumption billing applications, for example, it may be important to separately measure both, the fundamental and the total active power $\left(\mathrm{P}_{1}\right.$ and $\mathrm{P}$, respectively), as have been proposed to be a more fairest way to calculate the electrical energy consumed by a customer
[3]. In this regard, the proposed laboratory could be used as a tool, to experimentally evaluate the impact of different billing calculation approaches.

In order to achieve the goal of developing analytical and experimental skills between the students of universities of Strathclyde, UK, and Universidad Autonoma de Yucatan (UADY), Mexico, a set of laboratory experiments have been developed by collaborative efforts between aforementioned institutions.

The proposed Laboratory exercises are designed to be easily implemented by the students, using pre-existing Matlab Simulink blocks and also brings the opportunity to develop new measurements algorithms.

\section{Methodology}

The basic laboratory setup consists of an AC Power Supply, an electrical load(s), a metering device and a personal computer.

In this section, the characteristics of the different components of the laboratory are described, particularly, the metering device.

\section{A. Power Supply}

The metering device allows different AC power supply characteristics such as single or three phase, up to $240 \mathrm{~V}$ RMS (per phase) and a line frequency of 50 or $60 \mathrm{~Hz}$.

\section{B. Electrical load}

The electrical load could be any linear or non-linear load(s) connected to the power supply. The only constraint is the maximum current permitted by the current transducers.

\section{Metering device}

The metering device consists of a Raspberry Pi with an attached expansion module, used to safely measure voltage and current signals, called "Smartpi 2.0" [4]. The Raspberry $\mathrm{Pi}$ is connected via Ethernet to a $\mathrm{Pc}$, for the data analysis and visualization.

1) Smartpi 2.0: this electronic board is designed to function as a Smart Electricity Meter when is connected to a Raspberry Pi board. The voltage and current inputs are isolated, as well as the communication signals. 
a) Voltage and current inputs: the Smartpi is designed to work with current transformers with a secondary maximum output current of 1 Ampere. The voltage phases could be connected directly to the metering device.

b) Energy Metering IC: the Smartpi expansion module incorporates an ADE7878 Energy Metering IC [5], burden resistors for the CTs and resistive voltage dividers, in order to condition the analogue signals. Then, a DSP module inside the ADE7878 makes the calculations for relevant electrical quantities.

2) Raspberry P $i$ : for this laboratory, the Raspberry Pi is used to gather the data processed by the ADE7878 and also to perform different calculations, applied to the acquired voltage and current RMS values. The raw measurements of voltage and current waveforms cannot be obtained through the Smartpi board, because it only uses the $\mathrm{I}^{2} \mathrm{C}$ protocol to communicate with the processor. Nonetheless, these waveforms could be obtained directly from the ADC outputs of the ADE7878, via HSDC communication protocol, available on the ADE7878EBZ evaluation board [6].

\section{Personal Computer}

The Personal computer is used, in this laboratory, to configure and to program the Raspberry Pi. Although the code could be written in different languages such as $\mathrm{C} / \mathrm{C}++$, Phyton, etc., in this paper, the focus is on the Matlab Simulink code generation, for the sake of simplicity.

The personal computer is also used to visualize and log the data.

\section{PROPOSED EXERCISES}

The exercises proposed in this paper for the Power System Metrology Laboratory, are intended to demonstrate the capabilities of the system and are organized as follows:

\section{A. Basic Electrical Measurements accuracy}

Using the data calculated by the internal DSP of the ADE7878, the students will evaluate the accuracy of the measuring device with different types of loads (linear and non-linear), particularly, under non-sinusoidal conditions. Then, an analysis of the whole signal chain shall be done, in order to estimate the uncertainty contribution of the different components of the system (i.e. transducers, filters, etc.).

\section{B. Electric Power quantities definitions}

Different power definitions will be evaluated through this exercise. First, the students will refer to the datasheet of the ADE7878 to look at the formulas implemented by the integrated circuit. Then, using Simulink blocks, the students will implement formulas (defined by different authors) to evaluate the results. Such results are expected to be equivalent under sinusoidal conditions, but different, when reactive power is present in the electrical system.

\section{PMU algorithm}

Using the waveform values, obtained directly from the ADE7878 ADC's outputs, the Raspberry Pi performs as a PMU device, when a pre-existing Simulink algorithm is programmed on it. Such algorithm is intended to be a learning tool and not a fully functional PMU device. However, with the addition of an accurate clock reference, it is possible to be done.

\section{CONCLUSION}

A low-cost Power Systems Metrology Laboratory has been designed to facilitate the evaluation of the different subsystems included in modern measuring devices. A set of exercises has been proposed, with the focus on the analysis of the accuracy of electrical measuring devices, under distorted waveform conditions. The capabilities of the proposed Laboratory are not limited to those described in this document and could be easily escalated to more sophisticated projects or applications.

\section{ACKNOWLEDGEMENT}

Quijano Cetina R. wishes to express his gratitude to the Mexican Energy Ministry (SENER) and the National Council for Science and Technology (CONACYT) for financing this study through the Scholarship CONACYTSECRETARIA DE ENERGIA - SUSTENTABILIDAD ENERGETICA 2016- ref.: 291041/ 439171.

\section{REFERENCES}

[1] R. Quijano Cetina, A. J. Roscoe, and P. S. Wright, "Challenges for smart electricity meters due to dynamic power quality conditions of the grid: a review," 2017.

[2] "IEEE Standard Definitions for the Measurement of Electric Power Quantities Under Sinusoidal, Nonsinusoidal, Balanced, or Unbalanced Conditions," IEEE Std 1459-2010 (Revision of IEEE Std 1459-2000). pp. 1-50, 2010.

[3] A. J. Berrisford, "A Smarter Meter: IEEE-1459 power definitions in an off-the-shelf Smart Meter," 2015 IEEE International Instrumentation and Measurement Technology Conference (I2MTC) Proceedings. pp. 830-835, 2015.

[4] Enerserve, "SmartPi $2.0 \mid$ shop.enerserve.eu." [Online]. Available: https://shop.enerserve.eu/262/smartpi-2.0. [Accessed: 17-Jan-2018].

[5] “ADE7878 Poly Phase Multifunction Energy Metering IC with Total and Fundamental Powers.” Analog Devices.

[6] “ADE7878 Evaluation Board User Guide UG-146.”. 